\title{
Newer Insights in Early Childhood Dental Caries
}

\author{
Satarupa Ghosal ${ }^{1}$ and Sayan Bhattacharyya ${ }^{2 *}$ \\ ${ }^{1}$ Dental Surgeon, ECHS Polyclinic, Baruipur, West Bengal, India \\ ${ }^{2}$ Department of Microbiology, All India Institute of Hygiene and Public Health (AIIH\&PH), India
}

*Corresponding author: Sayan Bhattacharyya, Assistant Professor, Department of Microbiology, All India Institute of Hygiene and Public Health (AIIH\&PH), Kolkata, India

\begin{abstract}
Early dental caries is a common devastating disease of early childhood but can be prevented easily by simple methods. It is more common in developing countries and poor socioeconomic status but can be found in all settings. Different bacteria interacting with host salivary molecules, play a role in the development and aggravation of these lesions. Newer techniques for diagnosis, bacterial isolation, as well as treatment are coming up. Clinicians and researchers should be abreast with new developments to ensure proper diagnosis and treatment of these cases.
\end{abstract}

Keywords: Caries; sugar; cariogenic; streptococcus mutans

\section{Introduction}

Dental caries is a clinical challenge, especially in young children [1]. In fact, it is the commonest chronic infectious disease of childhood, and caused due to interaction or oral bacteria like Streptococcus mutans with sugary foods and saliva [1]. Of all dental caries, Early Childhood caries or ECC can be defined as "the presence of one or more decayed (non-cavitated or cavitated lesions), missing teeth (due to caries), or filled tooth surfaces in any primary tooth in a child 72 of months age or younger [1]. ECC is also called baby bottle-fed tooth decay, early childhood dental decay, comforter caries, nursing caries, maxillary anterior caries and rampant caries [1]. In children younger than 3 years of age, in addition, any sign of smooth-surface caries indicates severe early childhood caries (S-ECC) [1]. Early dental caries is frequently encountered in children of less than 71 months of age. Poor oral hygiene or insufficient dental plaque removal can lead to the rapid progression of ECC [2]. As regards bacterial etiology of dental caries, some strains, such as Streptococcus sanguinis, are reportedly associated with healthy teeth, while others, such as $S$. mutans, other oral Streptococcus spp., Veillonella spp., Actinomyces spp., Bifidobacterium spp., and Lactobacillus fermentum, were associated with caries [3]. There is also enough evidence that Gram positive bacteria like Actinomyces gerencseriae and other Actinomyces spp. play an important role in caries initiation [3]. In a study by Munson et al, in addition to S. Mutans and Lactobacillus spp., Rothia denticariosa and Propionibacterium acnes have also been found in caries lesions [4].

\section{Epidemiology}

ECC affects infants and preschool children worldwide, and its prevalence, though variable, can be up to $85 \%$ in disadvantaged groups [5]. Prevalence of ECC also varies widely, depending several variables like race, culture, and ethnicity, socioeconomic status, lifestyle, diet and oral hygiene practices and also according to the regional factors from country to country and from area to area [6]. A review of the literature suggests that in most developed countries the prevalence rate of ECC is between 1 and 12\% [6]. In less developed countries and among the disadvantaged or poorer groups in developed countries, the prevalence of ECC has been shown to be as high as 70\% [6]. However, it is not restricted to children with low socioeconomic status [2]. Recent data, for example, from Australia show a prevalence of ECC of more than $50 \%$ in 6-year-old children with caries on deciduous teeth [7]. Milsom et al. found that children with an already existing caries lesion have a 5-6 times higher incidence of developing new caries lesions compared to previously caries-free children [8]. Sleeping problems and insufficient sleep has also been identified as risk factor for ECC, because sleeping problems lead to more frequent use of night-time bottle use containing sugar-sweetened beverages 
[9]. There is a relationship with gender, since according to studies, the highest prevalence of ECC is found in the 3-4-year-old age group; also, boys are significantly more affected than girls, aged between 8 months and 7 years [10].

\section{Risk factors}

\section{Many risk factors have been identified like}

a) Most of the studies have shown significant correlation between ECC and bottle-feeding and sleeping of the baby with a bottle [1].

b) There is substantial evidence that prolonged and nocturnal breastfeeding is associated with an increased risk of ECC, particularly after the age of 12 months [1].

These aggravate caries due to less saliva production at night and less bacterial clearing.

c) Fermentable carbohydrates are a major factor in the development of dental caries. The small size of these sugar molecules allows salivary amylase enzyme to split the molecules into components that can then be easily metabolized by the bacteria in the plaque [11]. This process leads to bacteria producing acidic end products with subsequent demineralization of teeth.

d) Enamel hypoplasia due to premature birth, low birth weight or malnutrition is also a very important risk factor for caries development [12].

\section{Clinical features}

In the initial phases, ECC appears as a dull, white demineralized enamel that quickly progresses to obvious decay along the gingival margin [13]. Primary maxillary incisors are usually afflicted earlier than the four maxillary anterior teeth which are often involved simultaneously [1]. Carious lesions can be found on either the labial or lingual surfaces of the teeth and, sometimes on both [1]. The decayed hard tissue is clinically apparent as a yellow or brown cavitated area [1].

\section{Diagnosis}

Diagnosis is clinical. Culture of the bacteria can be carried out in Mitis-Salivations agar, from where colonies can further be identified [14]. However, S. mutans may be slightly inhibited in this medium and S. mitis may need longer incubation [14].

\section{Etiology and Pathogenesis}

It has been depicted by Corby et al that some bacteria are associated with healthy or caries -free teeth, like Streptococcus parasanguinis, Abiotrophia defectiva, Streptococcus mitis, Streptococcus oralis, and S. sanguinis [15]. The same group also showed that Actinomyces species, S. mutans, and Lactobacillus spp. were consistently associated with disease [15]. S. mutans and S. sobrinus have been recognized over the years as the main culprits behind development of Early childhood Dental caries [16]. They damage the dental enamel in presence of fermentable carbohydrates like Glucose, sucrose and fructose. In fact, S. mutant is present in about $30 \%$ of the plaques in carious teeth compared to $0.1 \%$ in healthy teeth [17]. Nowadays, it is well studied that not only bacteria, but also fungi, such as Candida albicans and the interactions between several different microbes, can enhance the progression of caries [18] . Bacteria and other microbes degrade sugars and lead to acid production which causes demineralisation of teeth and caries development [1]. Enamel of deciduous teeth is more vulnerable to acid-mediated damage than permanent teeth because it is thinner and built quickly in about 24 months by ameloblasts than that of permanent teeth which takes about 16 years to be built [19].

\section{Implications}

If left untreated, ECC, also called rampant caries, can lead to rapid and complete destruction of the crown [5]. Hence there is need of rapid and accurate diagnosis of the condition and adoption of suitable preventive measures.

\section{Newer developments}

New research has unearthed new mechanisms of pathogenesis in ECC. Bacterial biofilms are rapidly produced and are made of exopolymer matrix or EPS. This EPS is formed more on exposure to sucrose and fructose, and lead to further adhesion and colonization by cariogenic bacteria on surface of damaged teeth. This increased formation of biofilm biomass or "visible plaque", often found on the smooth surfaces of the children at risk of ECC shows the importance of EPS in the pathological process [20]. Research has also demonstrated a few biomarkers or host salivary molecules that affect ECC development. For example, host CSP-1 helps in adhesion of cariogenic bacteria to the enamel [20]. There are 2 types of Proline-rich glycoproteins in human saliva: acidic PRP and basic PRP. Basic PRP helps in ammonia production and neutralise sugar acids, whereas acidic PRPs bind strongly to teeth and enhance adhesion of the cariogenic bacteria [20]. These molecules can be biomarkers to help in assessing prognosis of ECC.

\section{Newer bacterial and other agents}

Atopobium vaginale has been found significantly in ECC in those cases that also grow Streptococcus mutans [3]. Bifidobacterium species have been associated with deep caries lesions [21]. In case of severe ECC, Porphyromonas catoniae has been found very commonly in the plaques [22]. Novel techniques like The Human Oral Microbe Identification Microarray (HOMIM) can be used for this type of assessment [22].

\section{Newer options for treatment}

Usually Children at low risk generally do not need any restorative therapy [1]. Children at moderate risk may require restoration of the progressing and cavitated lesions, while white spot and enamel proximal lesions should be treated by preventive techniques and then monitored for progression [1]. Children at high risk, however, may need earlier restorative interventions of enamel proximal lesions, and intervention of the progressing and cavitary lesions to minimize continual caries development [1]. Sometimes stainless-steel crown following pulpotomy and pulpectomy may be needed in cases of severe ECC [1]. 


\section{Prevention}

Prevention of ECC should focus on educating the parents about no nocturnal feeding and dietary modification [5]. Parents also should be advocated to maintain optimal dental health during pre- and postnatal periods [5]. Child health professionals, like physicians, assistants of physician, nursing practitioners, and nurses can play a very significant role in reducing the burden of this disease through monitoring, prompt diagnosis and health promotion activities [1]. Prevention of the progress of ECC can be carried out with the help of restorations, diet counselling, educating parents regarding decay-promoting feeding habits, maintenance of good oral hygiene, and the use of preventive agents like topical fluorides [5].

\section{Discussion}

ECC is a very common chronic disease of childhood and easily preventable by simple measures [5]. The associated pain from dental caries has got a negative impact on the child's emotional status, sleep patterns, and ability to learn or perform usual daily activities. A wide range of risk factors are linked with ECC in children from underprivileged and low socioeconomic status [5]. Oral health has been recognized as an essential prerequisite for general health and quality of life. Therefore, both oral disease prevention and oral health promotion should be included as integral components of chronic disease prevention and general health promotion programmes [5].

\section{Conclusion}

Early childhood caries should be diagnosed and treated early. New research is showing new avenues of diagnosis and aetiopathogenesis study.

\section{References}

1. Colak H, Dulgergill CH, Dalli M, Hamidi MM (2013) Early childhood caries update A review of causes diagnoses and treatments. J Nat Sci Biol Med 4(1): 29-38.

2. Meyer F, Enax J (2018) Early Childhood Caries Epidemiology Aetiology and Prevention. Hindawi International J Dentistry Article ID 1415873.

3. Aas JA, Griffen AL, Dardis SR, Lee AM, Olsen I, et al. (2008) Bacteria of Dental Caries in Primary and Permanent Teeth in Children and Young Adults. J Clin Microl 46(4):1407-1417.

4. Munson MA, Banerjee A, Watson TF, W G Wade (2004) Molecular analysis of the microflora associated with dental caries. J Clin Microbiol 42(7): 3023-3029.

5. Sukumaran A, Anand PS (2017) Early Childhood Caries Prevalence Risk Factors and Prevention. Front Pediatr 5: 157.
6. Congiu G, Campus G, Luglie PF (2014) Early childhood caries (ECC) prevalence and background factors A review. Oral Health Prev Dent 12(1): 71-76.

7. Chrisopoulos S, Harford JE (2016) Oral Health and Dental Care in Australia: Key Facts and Figures 2015. Australian Institute of Health and Welfare and the University of Adelaide, Canberra, ACT, Australia.

8. Milsom KM, Blinkhorn AS, Tickle M (2008) The incidence of dental caries in the primary molar teeth of young children receiving National Health Service funded dental care in practices in the North West of England. British Dental Journal 205(7): E14.

9. Shantinath SD, Breiger D, Williams BJ, Hasazi JE (1996) The relationship of sleep problems and sleep-associated feeding to nursing caries. Pediatric Dentistry 18(5): 375-378.

10. Ramos Gomez FJ, Weintraub JA, Gansky SA, Hoover CI, Featherstone JD (2002) Bacterial behavioral and environmental factors associated with early childhood caries. J Clin Pediatr Dent 26(2): 165-173.

11. Jensen ME (1999) Diet and dental caries. Dent Clin North Am 43: 615633.

12. Pitiphat W, Luangchaichaweng S, Pungchanchaikul P, Angwaravong 0 , Chansamak N (2014) Factors associated with molar incisor hypomineralization in Thai children. Eur J Oral Sci 122(4): 265-270.

13. Berkowitz RJ (2003) Causes treatment and prevention of early childhood caries A microbiologic perspective. J Can Dent Assoc 69(5): 304-307.

14. http://himedialabs.com/TD/M259.pdf. Last accessed 15/01/2020.

15. Corby PM, Lyons Weiler J, Bretz WA, Hart TC, Aas JA, et al. (2005) Microbial risk indicators in early childhood caries. J Clin Microbiol 43(11): 5753-5759.

16. Tanzer JM, Livingston J, Thompson AM (2001) The microbiology of primary dental caries in humans. J Dent Educ 65(10): 1028-1037.

17. Loesche WJ (1985) Nutrition and dental decay in infants. Am J Clin Nutr 41(2): 423-435.

18. Sztajer H, Szafranski SP, Tomasch J, Reck M, Nimtz M, et al. (2014) Crossfeeding and interkingdom communication in dual-species biofilms of Streptococcus mutans and Candida albicans. ISME Journal 8(11): 2256-2271.

19. Moll KJ, Moll M (2000) Kurzlehrbuch Anatomie, Elsevier, New York, NY, USA.

20. Karjalainen S, Soderling E, Sewon L, Lapinleimu H, Simell O (2001) A prospective study on sucrose consumption, visible plaque and caries in children from 3 to 6 years of age. Community Dent Oral Epidemiol 29(2): 136-142.

21. van Houte J, Lopman J, Kent R (1996) The final pH of bacteria comprising the predominant flora on sound and carious human root and enamel surfaces. J Dent Res 75(4):1008-1014.

22. Ma C, Chen F, Zhang Y, Sun X, Tong P, et al. (2015) Comparison of Oral Microbial Profiles between Children with Severe Early Childhood Caries and Caries-Free Children Using the Human Oral Microbe Identification Microarray. Plos One 10(3): e0122075. 
(C) (i) This work is licensed under Creative Commons Attribution 4.0 License

To Submit Your Article Click Here: Submit Article

DOI: $10.32474 /$ IPDOAJ.2020.03.000170

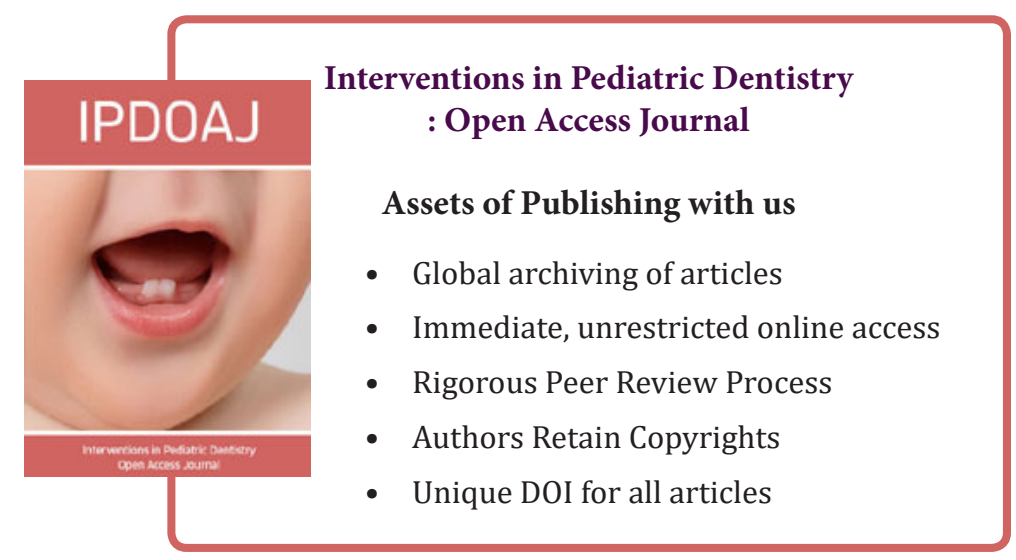

